\title{
Los Dominios de la Personalidad y su Relación con el Estilo de Liderazgo Transformacional
}

\author{
Danny Arévalo-Avecillas ${ }^{(1) \star}$, Carmen Padilla-Lozano(1), Ricardo Pino ${ }^{(2)}$ y Holger Cevallos $^{(3)}$ \\ (1) Universidad Católica de Santiago de Guayaquil, Km 1⁄2 vía Carlos Julio Arosemena, Guayaquil, Ecuador \\ (e-mail: econ.darevalo@gmail.com, carmen.padilla@cu.ucsg.edu.ec) \\ (2) CENTRUM Católica Graduate Business School, Jr. Daniel Alomía Robles 125, Urb. Los Álamos de Monterrico, \\ Pontificia Universidad Católica del Perú, Lima, Perú (e-mail: rpino@pucp.pe) \\ (3) Escuela Superior Politécnica del Litoral, Facultad de Ciencias Naturales y Matemáticas, Campus Gustavo Galindo \\ Km. 30.5 Vía Perimetral, P.O. Box 09-01-5863, Guayaquil, Ecuador (e-mail: holgceva@espol.edu.ec)
}

${ }^{*}$ Autor a quien se debe dirigir de correspondencia

Recibido Nov. 27, 2018; Aceptado Ene. 16, 2019; Versión final Feb. 22, 2019, Publicado Jun. 2019

\begin{abstract}
Resumen
Se evalúa el impacto de los dominios de la personalidad y la experiencia de trabajo en el estilo de liderazgo transformacional, entregando nuevas evidencias de la relación causal entre ambos constructos. La investigación tuvo un enfoque cuantitativo con un diseño de investigación no experimental, de corte transversal y de alcance correlacional-causal. Se realizó una encuesta a 368 profesionales que cursan programas de posgrado en Administración de Empresas en el Ecuador. El instrumento denominado Inventario Revisado de Personalidad se utilizó para medir los cinco dominios de la personalidad: (a) extraversión, (b) amabilidad, (c) escrupulosidad, (d) neuroticismo, y (e) apertura a la experiencia. Por otra parte, el Cuestionario Multifactorial de Liderazgo (MLQ) fue el instrumento utilizado para medir el estilo de liderazgo transformacional. Para el análisis de datos se utilizó análisis de correlaciones y un modelo de regresión multivariado. Los resultados muestran que los dominios extraversión y escrupulosidad fueron los más importantes en la proyección del estilo de liderazgo transformacional.
\end{abstract}

\section{Personality Domains and their relationship with the Transformational Leadership Styles}

\begin{abstract}
The impact of personality domains and work experience in the transformational leadership style, providing new evidence of the causal relationship between both constructs is evaluated. The research had a quantitative approach with a no-experimental design, cross-sectional and correlational- causal scope. A survey was applied to 368 professionals of several Master of Business Administration programs in Ecuador. The instrument called Revised Personality Inventory was used to measure the five domains of personality: (a) extraversion, (b) agreeableness, (c) conscientiousness, (d) neuroticism, and (e) openness to experience. On the other hand, the Multifactor Leadership Questionnaire (MLQ) was the instrument used to measure the transformational leadership style. To analyze the data correlation analysis and a multivariate regression model were used. The results highlight that extraversion and conscientiousness domains proved to be the most important in the projection of transformational leadership style.
\end{abstract}




\section{INTRODUCCIÓN}

Determinar la relación entre personalidad y liderazgo continúa atrayendo atención entre los investigadores. La mayoría de los estudios de personalidad y liderazgo fueron desarrollados en Estados Unidos, y pocos otros en países como Noruega, Australia, Singapur, y Canadá, pero casi ningún estudio similar se ha efectuado en un país en desarrollo (Bass, 2008; Bono y Judge, 2004; Bono et al., 2012; Crossan et al., 2012). D’Alessio (2008) realizó un estudio para replicar la investigación sobre la personalidad y su influencia en el liderazgo para el caso peruano, utilizando una muestra de 500 administradores que cursaban la Maestría en Administración de Negocios (MBA). D'Alessio concluyó que los resultados proveían evidencia de que la experiencia de trabajo desempeña un rol importante en el desarrollo de los comportamientos del liderazgo. Además, la relación funcional entre la personalidad y el liderazgo mantiene ciertas similitudes a nivel de resultados con trabajos previamente llevados a cabo en países desarrollados; no obstante, es importante considerar las diferencias intrínsecas generadas por el entorno global y que afectan de manera directa al comportamiento general de los empresarios, administradores y otros tomadores de decisiones. Torres y Jones (2010), partiendo de los resultados de las investigaciones que realizara Hofstede $(2001 ; 2006)$ respecto a las diferencias culturales que pueden identificarse entre empresarios de diferentes países, realizaron una actualización y contraste de dichos resultados aplicados para los empresarios de Perú y Países Bajos. Los resultados revelaron que, efectivamente, se mantenían diferencias entre ciertas características derivadas del entorno cultural y que en el ámbito empresarial existen condiciones intrínsecas que se manifiestan claramente en el comportamiento de los individuos en términos generales, y por ende se manifestarán de igual manera en el desempeño de sus actividades, ya sean estas al emprender o administrar un negocio.

La evidencia empírica sugiere que existe una relación entre los diferentes rasgos de personalidad que manifiestan los individuos y la propensión que estos demuestran hacia manifestar o desarrollar estilos de liderazgo (Bono y Judge, 2004; D'Alessio, 2008). La discusión al respecto aún es amplia, principalmente considerando la disyuntiva entre si el liderazgo es un rasgo nato y propio del individuo, o si es un rasgo que puede desarrollarse (Bass, 2008; Lim y Ployhart, 2004). No obstante, la definición de ciertas características en cómo puede manifestarse el liderazgo, no permite resolver de manera directa un cuestionamiento básico pero primordial respecto a qué define a un gran líder. Para establecer una conceptualización que permita predecir un estilo de liderazgo a manifestarse en función de otros rasgos de los individuos, Judge et al. (2002) plantearon evaluar la posible relación que pudiera existir entre los componentes de la personalidad de los individuos y el estilo de liderazgo que estos pueden manifestar.

Existe un debate histórico sobre la delimitación de los rasgos de la personalidad, partiendo desde Aristóteles quien clasificó los temperamentos de los individuos en varias categorías generales (Judge y Bono, 2000). Por ello, antes de analizar su vinculación con el liderazgo, cabe examinar a profundidad los elementos que componen al inicialmente conocido como Los Cinco Grandes y posteriormente popularizado a inicios de los noventa como el Modelo de los Cinco Factores de Personalidad (Costa y McCrae, 1992). La investigación de la personalidad en psicología reconoce una hipótesis léxica que afirma que las características de la personalidad más importantes en un individuo eventualmente se convertirán en parte de su lenguaje y mientras más importantes sean estas características, es más probable que se codifiquen en el lenguaje con una sola definición (Tanasescu et al., 2013). Es así como interviene la hipótesis léxica para poder reconocer cinco grandes dominios o dimensiones de la personalidad, donde sus descriptores se agruparon después del análisis factorial. Sin embargo, se tiene que por varios años los investigadores estudiaron el mismo fenómeno en estructuras de cinco factores, pero bajo distintos parámetros, considerando diferentes instrumentos, descripciones y conceptualizaciones, tales como Golderg (1990). Aquello generó un cuestionamiento y una crítica al modelo de Los Cinco Grandes, pues su estudio variaba significativamente en su interpretación según su autor, por lo que se consideró que el término Los Cinco Grandes era poco preciso y descriptivo y difícilmente expresaba la gama completa de rasgos de personalidad. Consecuentemente, Costa y McCrae (1992) propusieron una alternativa, denominarlo como El Modelo de los Cinco Factores (FFM por sus siglas en inglés) que proporciona una evaluación profunda y completa de la personalidad adulta (D'Alessio, 2008) pero sin descartar la estructura tradicional de los cinco factores ya reconocidos.

El instrumento desarrollado por Costa y McCrae (1992) para medir el modelo se denomina Inventario Revisado de Personalidad NEO PI-R, el cual se basa en estudios factor-analíticos de la estructura de la personalidad que contiene 30 facetas organizadas en cinco dominios: (a) extraversión, extrovertido o enérgico vs solitario o reservado; (b) amabilidad, amigable o compasivo vs frío o poco amable; (c) escrupulosidad, eficiencia u organización vs despreocupado o descuidado; (d) neuroticismo, sensible o nervioso vs seguro o confiable; y (e) apertura a la experiencia, inventivo o curioso vs consistente o cauteloso (Tanasescu et al., 2013). Este modelo ha sido considerado como uno de los más exhaustivos en la medición de la personalidad, por lo que, a pesar de que existen otros modelos que se derivan del mismo, los estudios de la personalidad se han estandarizado con el uso del FFM (McAdams y Donnellan, 2009). 
En el análisis individual de los dominios se destaca que las personas con alta extraversión se consideran bastante sociables, pues pueden dar uso inclusive de su sentido del humor, capacidad de introducción de temas de conversación y de estimulación de la interacción social. Son personas en definitiva sociables y gregarias (Do y Minbashian, 2014). Por otra parte, una personalidad de alta amabilidad se destaca por ser confiable, altruista, tener tacto y sensibilidad; refleja una tendencia a ser cálido y honesto y representa una predisposición hacia la cooperación, cortesía y valoración de la afiliación mientras evita el conflicto (Bass, 2008; D'Alessio, 2008). Los rasgos que se asocian con un alto grado de amabilidad incluyen la cortesía, flexibilidad, generosidad y tolerancia. Esta personalidad sugiere que el individuo se distingue como una persona simpática desde la perspectiva de quienes le rodean, además de que se encuentra normalmente dispuesta a ayudar a los demás pues cree que su buena actitud será recíproca, por lo que son sensibles ante las necesidades de los demás (Arora y Rangnekar, 2016). Un alto grado de escrupulosidad destaca el esfuerzo orientado al éxito del individuo y se admira su responsabilidad, gran sentido de dirección y compromiso por el trabajo duro para alcanzar objetivos (Costa y McCrae, 1992). Son personas que se caracterizan por su puntualidad y confiabilidad y de allí radica la importancia en el estudio de los rasgos de la psicología laboral (Bono y Judge, 2004). Los individuos escrupulosos se consideran como poseedores de una personalidad con iniciativa, disciplina, persistencia y organización, así como con gran dedicación y motivación (Zopiatis y Constanti, 2012). Un bajo puntaje de escrupulosidad no implica una carencia de valores, pero sí una aplicación poco eficiente de los mismos pues tienden a ser hedonistas al esforzarse menos por alcanzar sus metas (D'Alessio, 2008).

Con respecto al neuroticismo, una alta calificación puede afectar la estabilidad y desempeño del individuo a causa de un nerviosismo y hostilidad crónica; inclusive se ha encontrado una fuerte relación entre el neuroticismo con la baja autoestima, baja confianza en sí mismo y baja autoeficacia general (Costa y McCrae, 1992; Judge et al., 2002). No obstante, todas las personas pueden ser figuradas con un determinado nivel de confianza, indistintamente de la calificación que obtengan en las dimensiones o dominios de personalidad del modelo (Tanasescu et al., 2013). Por su parte, los individuos con bajo neuroticismo reflejan ser más calmados y relajados. También se ha encontrado que existe una correlación que indica que las personas con altos niveles de bienestar poseen alta calificación en extraversión, amabilidad y escrupulosidad, pero una baja calificación de neuroticismo (Soto, 2015). Bono y Judge (2004) indican que las conceptualizaciones tradicionales de la apertura a la experiencia hacen énfasis en la cultura y el intelecto. La cultura corresponde a la apreciación por las artes y la ciencia, mientras que el intelecto corresponde a la habilidad de aprender y razonar, motivo por el que los individuos con alto grado de este dominio son emocionalmente sensibles e intelectualmente curiosos (Kaufman et al., 2016). Un alto grado de intelecto, cultura o de apertura a la experiencia, denota que el individuo tiene un amplio rango de intereses, por lo que tiende a vincular sus prioridades con la versatilidad de su entorno al tomar una actitud crítica frente a la sociedad (Bono y Judge, 2004; D'Alessio, 2008).

La investigación sobre el liderazgo halla uno de sus principales referenciales en el trabajo de Burns (1978) a quien se le atribuye la conceptualización del denominado liderazgo transformacional, conocido también como liderazgo carismático. Según Burns, el liderazgo puede diferenciarse entre transformacional y transaccional. Un líder que refleja liderazgo transformacional obtiene apoyo inspirando a sus seguidores a identificar una visión que permita ir más allá de los intereses inmediatos propios de los seguidores, mientras que un líder transaccional busca la cooperación de los seguidores planteando un intercambio para luego proceder a evaluar la relación de intercambio planteada. No obstante, existen autores como Bass (2008) quienes plantean que los líderes pueden desempeñar un liderazgo transaccional y transformacional a la vez, incluso ninguno. Es importante acotar que el liderazgo puede llevar a las empresas a generar ventaja competitiva sostenida a mediano y largo plazo, a través de una óptima cultura organizacional que se sustente en la innovación y el desarrollo tecnológico sostenible (Crossan et al., 2012).

Gran parte de la literatura de los estilos de liderazgo se centra en el transaccional y el transformacional (Zareen et al., 2015). De igual forma, ambos estilos de liderazgo han ayudado a predecir la satisfacción de los subordinados con sus líderes. No obstante, ambos no siempre pueden proveer una verdadera satisfacción al subordinado y parcialmente contribuyen como variables ilustrativas. Cabe destacar que adoptar cualquiera de los dos estilos puede ayudar a llevar a una compañía al éxito (Araneda et al., 2016). Los líderes transformacionales inspiran confianza, respeto y admiración por parte de sus seguidores, ya que promueven la resolución de problemas al vincularse con cada colaborador o subordinado de forma individual (BallesterMiquel et al., 2017). Los líderes transformacionales valoran el desempeño por encima de cualquier expectativa previa que tengan de un individuo (Bass y Avolio, 1994). Este estilo de liderazgo se centra en la transformación de los valores y creencias de sus seguidores mediante la inspiración, es decir, se nutre de una mutua estimulación y elevación que convierte a los seguidores en líderes y que también puede convertir a los líderes en agentes morales (Johnson y Dipboye, 2008; Spinelli, 2006). Los líderes transformacionales se distinguen de otros estilos porque en lugar de brindar directrices rígidas, el líder transformacional trata de explotar y desarrollar todo el potencial de sus seguidores (Bass, 2008) mediante la simpatía, lo que provoca en los 
seguidores un sentimiento de satisfacción y motivación, que se traduce en una percepción de crecimiento, transformación y realización organizacional, y se conoce como efecto de aumento (Bass, 2008).

Las dimensiones del liderazgo transformacional son: (a) influencia idealizada, correspondiente al carisma que produce el modelo a seguir por identificación de atributos y de comportamiento, desarrollando una gran aprobación sobre su accionar y una supresión de la crítica; (b) motivación por inspiración, que trata la habilidad de transmitir con compromiso y emoción una determinada visión del futuro; (c) estimulación intelectual, que corresponde a brindarle a los seguidores un enfoque nuevo a los problemas y un cuestionamiento de las suposiciones, propios valores y creencias; y (d) consideración individual, que refleja el comportamiento individual del líder frente a sus seguidores, tanto de mentor como de empleador (Bass, 2008; D'Alessio, 2008).

\section{OTROS ANTECEDENTES E HIPÓTESIS}

Han existido muchos avances en cuanto al estudio del liderazgo en todas las evoluciones de sus dimensiones, siendo el liderazgo transformacional el que más ha llamado la atención en las últimas décadas (Bass y Avolio, 1994; Boe y Holth, 2015; Bono et al., 2012; Judge y Bono, 2000). A pesar de toda la evidencia empírica, aún se mantienen preguntas como qué determina o predice al liderazgo transformacional (Lim y Ployhart, 2004). Mientras existen estudios que vinculan la personalidad con el liderazgo transformacional, la mayor parte de la investigación ha utilizado variados tipos de rasgos de personalidad, por lo que se complica la integración de dichos resultados (Bass, 2008; Furnham y Crump, 2015). Bass (2008) indicó que el apoyo empírico entre la relación del liderazgo transformacional y la personalidad es irregular, en especial si los resultados empíricos no guían hacia una conclusión clara. Son los meta-análisis los que podrían brindar una contribución valiosa, pero estos resultados también han traído consigo resultados débiles (Lim y Ployhart, 2004). Jude y Bono (2000) vincularon directamente al modelo de lo cinco factores con el liderazgo transformacional, encontrando que la extroversión (correlación de 0.28) y la amabilidad (correlación de 0.32) predecían positivamente al liderazgo transformacional. A pesar de la pequeña y moderada relación encontrada, Judge y Bono brindaron una evidencia preliminar de que ciertos rasgos del modelo de cinco factores podrían estar relacionados con el liderazgo transformacional, pues indican que la extraversión, la amabilidad y la apertura a la experiencia estarían positivamente relacionados con este liderazgo. Por ello se requiere de mayor estudio sobre el tema.

Judge et al. (2002) notaron que, del modelo de los cinco factores, más del $90 \%$ de las correlaciones individuales entre el neuroticismo, escrupulosidad, extraversión y apertura a la experiencia con el liderazgo, eran mayores a cero. También, señalaron que dicho modelo explica el $28 \%$ de la variabilidad entre las calificaciones de emergencia del liderazgo y el $15 \%$ de la variabilidad entre las calificaciones de efectividad del liderazgo (Bono y Judge, 2004). Por su parte, Lim y Ployhart (2004) encontraron que la extraversión y la amabilidad se relacionan significativamente con el liderazgo transformacional, cuya naturaleza de estudio consideró una muestra principalmente joven y completamente masculina. Se ha reconocido, además, una necesidad de enfrentar los retos ejecutivos mediante el desarrollo de líderes talentosos dentro de la compañía, para que la misma se vuelva exitosa. Algunos autores reconocen a este proceso como desarrollo de recurso o Resource Development (Crossan et al., 2012; McCall Jr y McCauley, 2014). El desafío dentro de las empresas es reconocer a las personas con mayor potencial y enviarlos a la acción, resultando en dos logros: resolviendo el problema organizacional y desarrollando un administrador mediante la experiencia (Hezlett, 2016). Según McCall Jr y Hollenbeck (2002), la experiencia como tal no tiene garantías de desarrollo de un líder determinado pues existen personas que no poseen apertura para aprender de sus experiencias. A pesar de esta aclaración, los autores proponen catalizadores de desarrollo como retroalimentación de varias fuentes, responsabilidad para el desarrollo, entrenamientos, metodología de desarrollo de prácticas deliberadas y sesiones de grupo. De allí que el estudio de Spinelli (2006) sugiriera incentivar la formulación e implementación de programas de entrenamiento gerencial para alcanzar un estilo de liderazgo transformacional. Por esta consideración, resulta necesario contar con un análisis detallado sobre la importancia de la experiencia de trabajo. D’Alessio (2008) indicó que el comportamiento de liderazgo transformacional está directamente relacionado con el incremento de la experiencia de trabajo y, por ende, con mayor edad.

El propósito de la presente investigación consistió en entregar nuevas evidencias sobre el impacto de los dominios de la personalidad y la experiencia de trabajo en el estilo de liderazgo transformacional para el contexto ecuatoriano. Considerando que la presente investigación recoge las experiencias de investigaciones previas que no han demostrado consistencia en la definición de una relación funcional entre los rasgos de personalidad y los comportamientos de liderazgo, se asume como hipótesis la existencia de esta relación funcional, establecida de la siguiente manera: $\mathrm{H}_{1}$ : Existe una relación funcional entre los rasgos de personalidad, como un constructo o conjunto de rasgos, y los comportamientos de liderazgo transformacional y transaccional. 
Para definir la validez de $\mathrm{H}_{1}$ se utilizó como referencia las relaciones funcionales. Las sub-hipótesis propuestas para el entorno ecuatoriano son: (a) $\mathrm{H}_{1.1}$ : Existe una relación negativa entre el neuroticismo y el liderazgo transformacional; (b) $\mathrm{H}_{1.2}$ : Existe una relación positiva entre la extraversión y el liderazgo transformacional; (c) $\mathrm{H}_{1.3}$ : Existe una relación positiva entre la apertura a la experiencia y el liderazgo transformacional; (d) $\mathrm{H}_{1.4}$ : Existe una relación positiva entre la amabilidad y el liderazgo transformacional; (e) $\mathrm{H}_{1.5}$ : Existe una relación positiva entre la escrupulosidad y el liderazgo transformacional; (f) $\mathrm{H}_{1.6}$ : Existe una relación positiva entre la experiencia laboral y el liderazgo transformacional; y $(\mathrm{g}) \mathrm{H}_{1.7}$ : Los cinco dominios de la personalidad, es decir, neuroticismo, extraversión, apertura a la experiencia, amabilidad, y escrupulosidad, más la experiencia de trabajo ejercen una influencia combinada sobre el estilo de liderazgo transformacional.

\section{METODOLOGÍA}

La presente investigación tuvo un enfoque cuantitativo, con un diseño de investigación no experimental, de corte transversal y de alcance correlacional - causal. Como fuente de información primaria se planteó la realización de encuestas luego de haber validado los instrumentos de medición de los rasgos de la personalidad y el estilo de liderazgo transformacional. Los instrumentos empleados para la medición de ambos constructos han sido validados y utilizados ampliamente en estudios previos con resultados satisfactorios. La población de estudio correspondió a los profesionales que cursan programas de posgrado en Administración de Empresas en el Ecuador. De acuerdo a información proporcionada por el Sistema Nacional de Información de Educación Superior del Ecuador (SNIESE, 2017), referente a la oferta académica, en Ecuador existen 19 programas de posgrado en Administración de Empresas y 8,772 estudiantes matriculados. Geográficamente, el $68 \%$ de dichos programas estaban concentrados en las provincias de Guayas y Pichincha. A partir de esta información se calculó la muestra, considerando un universo finito, dando como resultado 368 encuestas con un nivel de $95 \%$ de confianza. Cabe destacar que únicamente las universidades de categoría A y B acreditadas por el Consejo de Evaluación Acreditación y Aseguramiento de la Calidad pueden ofertar programas de maestría en el Ecuador.

Para la medición de los dominios de la personalidad como variables exógenas del modelo, se utilizó el instrumento de los cinco factores de personalidad desarrollado por Costa y McCrae (1992). Este instrumento, denominado Inventario Revisado de Personalidad NEO PI-R, se fundamenta en estudios de análisis factorial de la estructura de la personalidad y contiene 30 facetas organizadas en cinco dominios específicos: (a) extraversión; (b) amabilidad; (c) escrupulosidad; (d) neuroticismo; y (e) apertura a la experiencia (Tanasescu et al., 2013). Las facetas para cada dimensión son las siguientes: (a) Neuroticismo: ansiedad, hostilidad, depresión, autoconciencia, impulsividad y vulnerabilidad; (b) Extraversión: cordialidad, gregarismo, asertividad, actividad, búsqueda de emociones y emociones positivas; (c) Apertura a la experiencia: fantasía, estética, sentimientos, acciones, ideas y valores; (d) Amabilidad: confianza, franqueza, altruismo, conformidad, modestia y sensibilidad a los demás y (e) Escrupulosidad: competencia, orden, sentido del deber, necesidad de logro, autodisciplina y deliberación (D'Alessio, 2008). Cada faceta se mide con ocho ítems, por lo que cada constructo contiene 48 ítems, dando un total de 240 preguntas que evalúan los rasgos de personalidad de una persona. Cada ítem se mide mediante una escala Likert de cinco puntos, que varía desde 1, muy en desacuerdo, hasta 5, muy de acuerdo.

Por otro lado, Bass y Avolio (1994) desarrollaron el instrumento denominado Multifactor Leadership Questionnaire (MLQ), muy utilizado en el campo de la psicología organizacional, que incluye una gama completa de medición del liderazgo. El presente estudio tomó los reactivos que miden el estilo de liderazgo transformacional con sus principales componentes, por medio de 20 preguntas que evalúan las características principales de este estilo de liderazgo. Los componentes del liderazgo transformacional son: (a) atributos de influencia idealizada; (b) comportamientos de influencia idealizada; (c) motivación inspiradora; (d) estimulación intelectual; $y$ (e) consideración individual. Cada ítem se mide mediante una escala Likert de cinco puntos, que varía desde 1, definitivamente no, hasta 5 , frecuentemente o casi siempre. El estudio contempla además la inclusión de la variable experiencia laboral, medida por el número de años que el participante ha trabajado durante su etapa profesional. El proceso de análisis de datos inició con una exploración de los datos para detectar datos ausentes y valores atípicos. Posteriormente, el proceso siguió la secuencia a continuación: (a) estadística descriptiva, (b) prueba de fiabilidad, (c) prueba de validez por medio de análisis factorial, (d) matriz de correlaciones y (e) modelo de regresión. Se obtuvo un resumen de los resultados de estadística descriptiva para los cinco dominios de la personalidad y los componentes del liderazgo transformacional. Por otra parte, el coeficiente alfa de Cronbach fue calculado para cada una de las 30 facetas de los cinco dominios de la personalidad y los componentes del estilo de liderazgo transformacional, con la finalidad de determinar la consistencia interna de las escalas. El análisis factorial exploratorio fue empleado tanto para el NEO-PIR como para el instrumento de medición del liderazgo transformacional, con el propósito de evaluar la estructura interna de ambos modelos y determinar si las 30 facetas explican adecuadamente los cinco dominios de la personalidad y si los cinco rasgos del liderazgo explican el estilo de liderazgo transformacional. 
Las relaciones entre la personalidad y el estilo de liderazgo transformacional fueron obtenidas a través del cálculo del coeficiente de correlación de Pearson. Se procedió a analizar la magnitud y la significancia de los coeficientes de correlación para evaluar las hipótesis planteadas sobre la asociación entre variables. De igual modo, se incluyó un modelo de regresión múltiple para evaluar las relaciones entre los dominios de personalidad, experiencia laboral y el liderazgo transformacional, siendo esta última la variable dependiente del modelo propuesto. Las variables independientes fueron: (a) neuroticismo, (b) extraversión, (c) apertura a la experiencia, (d) amabilidad, (e) escrupulosidad y (f) experiencia laboral. La prueba $F$ fue obtenida para decidir si el modelo presentaba una capacidad predictiva significativa estadísticamente. El coeficiente de determinación ajustado $R^{2}$ permitió evaluar el ajuste de los datos y los coeficientes de regresión informaron el efecto explicativo de cada variable independiente sobre la variable dependiente. Los resultados fueron procesados empleando SPSS 22 y AMOS 22.

\section{RESULTADOS}

La edad de los participantes de la muestra osciló entre 23 y 57 años y con un promedio de 34 años. El $70.65 \%$ de los participantes fueron de género masculino y el $29, .5 \%$ restante de género femenino. Además, existió un predominio de participantes graduados de carreras de pregrado como economía, administración y afines (56.25\%), en comparación con los graduados en carreras de ingeniería (27.99\%) y de otras carreras (15.76\%). Por último, los estudiantes indicaron que poseen un promedio aproximado de nueve años de experiencia profesional, y predominaron aquellos con experiencia laboral entre 10 y 13 años (37.23\%).

\section{Dominios de la Personalidad}

Los resultados de estadística descriptiva derivados del cuestionario NEO-PI-R en su versión en español se reportan en la Tabla 1. Por medio del cálculo de la media, desviación estándar y asimetría se destaca que la distribución de cada dominio fue aproximadamente simétrica. Es importante destacar que se presentan los resultados de los dominios, y no de las facetas que conforman cada dominio, debido a que la prueba utilizada en el presente estudio proporcionó un puntaje final para cada dominio. Este se compone de la suma de las puntuaciones totales de los ítems de cada faceta que conforman el dominio. Por tanto, estos valores oscilan entre 48 y 320 . El neuroticismo fue el dominio que presentó la puntuación más baja de tendencia central, con una media de 71.90 y dispersión, con un coeficiente de variación de 0.13 . Por otro lado, la escrupulosidad y extraversión fueron los dominios que exhibieron las puntuaciones más altas de tendencia central, con una media de 154.43 y 141.83 respectivamente; no obstante, la extraversión presentó una variación ligeramente superior a los datos del dominio de escrupulosidad. La amabilidad fue el dominio con mayor variabilidad de acuerdo a su coeficiente de variación de 0.22 .

Tabla 1: Estadística Descriptiva de los Dominios de Personalidad

\begin{tabular}{|l|c|c|c|c|c|c|c|}
\hline Dominios de Personalidad & Media & D.E. & Mínimo & Máximo & Asimetría & Curtosis & $\begin{array}{c}\text { Coef. } \\
\text { Variación }\end{array}$ \\
\hline Neuroticismo & 71.90 & 9.03 & 51 & 97 & 0.19 & -0.23 & 0.13 \\
\hline Extraversión & 141.83 & 25.46 & 62 & 170 & -1.24 & 0.84 & 0.18 \\
\hline Apertura a la experiencia & 119.09 & 21.68 & 67 & 158 & -0.42 & 0.52 & 0.18 \\
\hline Amabilidad & 128.89 & 28.51 & 60 & 164 & -0.70 & -0.42 & 0.22 \\
\hline Escrupulosidad & 154.43 & 19.66 & 107 & 183 & -0.55 & -1.07 & 0.13 \\
\hline
\end{tabular}

Posteriormente se obtuvo la medida de adecuación muestral de Kaiser-Meyer-Olkin (KMO) de 0.837 y la prueba de esfericidad de Bartlett con un estadístico $x^{2}$ de $7610.48(p<0.01)$. La regla establece que los valores de KMO por encima de 0.7 determinan una mejor adecuación de los datos a un modelo factorial. Por otro lado, la hipótesis alternativa de la prueba de esfericidad de Bartlett indicó que es posible aplicar el análisis factorial (Thompson, 2004). Dado que ambas condiciones se cumplieron, se concluyó que el uso del análisis factorial exploratorio (AFE) era apropiado.

Los factores se obtuvieron con el método de extracción, comúnmente utilizado, de análisis de componentes principales (Hair et al., 1999). De acuerdo con este método, cinco son los valores propios mayores que 1, que varían de 3.356 a 4.886 y que explican el $68,83 \%$ de la varianza total. Un análisis factorial exploratorio fue realizado para validar la replicabilidad del modelo de cinco factores del instrumento NEO-PI-R (ver Tabla 2). Las cargas factoriales de cada faceta con respecto a las dimensiones subyacentes fueron obtenidas a través de la rotación varimax de la matriz de la solución factorial en forma ortogonal. En esta rotación se destaca que la varianza total explicada no cambia y los factores no están correlacionados. 
Tabla 2: Cargas Factoriales de los Dominios de Personalidad

\begin{tabular}{|c|c|c|c|c|c|c|c|}
\hline \multirow{2}{*}{ Factor } & \multicolumn{5}{|c|}{ Factor } & \multirow[t]{2}{*}{$\alpha$ de Cronbach } & \multirow{2}{*}{$\begin{array}{l}\text { Varianza } \\
\text { explicada }\end{array}$} \\
\hline & $N$ & $E X$ & $A E$ & $A$ & $E S$ & & \\
\hline Dominio de neuroticismo & & & & & & 0.894 & $13.22 \%$ \\
\hline N1. Ansiedad & .917 & -.002 & .083 & -.043 & .016 & 0.419 & \\
\hline N2. Hostilidad & .763 & .015 & .020 & .023 & .029 & 0.199 & \\
\hline N3. Depresión & .854 & .020 & .050 & .017 & .035 & 0.287 & \\
\hline N4. Autoconciencia & .702 & .028 & -.070 & .030 & .003 & 0.333 & \\
\hline N5. Impulsividad & .769 & .022 & .023 & -.024 & -.018 & 0.232 & \\
\hline N6. Vulnerabilidad & .832 & .002 & .063 & .022 & -.002 & 0.295 & \\
\hline Dominio de extraversión & & & & & & 0.905 & $13.68 \%$ \\
\hline EX1. Cordialidad & -.048 & .783 & .080 & -.037 & .024 & 0.646 & \\
\hline EX2. Gregarismo & .031 & .808 & -.006 & -.063 & .041 & 0.715 & \\
\hline EX3. Asertividad & .024 & .828 & .014 & -.026 & .055 & 0.691 & \\
\hline EX4. Actividad & .016 & .818 & .053 & -.016 & -.028 & 0.630 & \\
\hline EX5. Búsqueda de emociones & .042 & .861 & -.008 & .018 & .043 & 0.676 & \\
\hline EX6. Emociones positivas & .031 & .835 & -.022 & .019 & .032 & 0.645 & \\
\hline \multicolumn{2}{|l|}{ Dominio de apertura a la experiencia } & & & & & 0.920 & $14.44 \%$ \\
\hline AE1. Fantasía & .027 & .031 & .850 & .045 & -.003 & 0.687 & \\
\hline AE2. Estética & .036 & .042 & .841 & .002 & -.026 & 0.707 & \\
\hline AE3. Sentimientos & -.005 & .008 & .852 & .029 & .029 & 0.662 & \\
\hline AE4. Acciones & .059 & -.017 & .809 & .002 & -.052 & 0.668 & \\
\hline AE5. Ideas & .030 & .053 & .839 & .026 & -.019 & 0.662 & \\
\hline AE6. Valores & .005 & -.002 & .876 & .018 & .048 & 0.614 & \\
\hline Dominio de amabilidad & & & & & & 0.945 & $15.81 \%$ \\
\hline A1. Confianza & .060 & -.077 & .040 & .890 & .053 & 0.768 & \\
\hline A2. Franqueza & -.079 & .061 & .015 & .853 & -.020 & 0.731 & \\
\hline A3. Altruismo & .025 & -.032 & -.015 & .893 & -.017 & 0.811 & \\
\hline A4. Actitud conciliadora & .014 & .006 & .045 & .886 & .032 & 0.761 & \\
\hline A5. Modestia & -.026 & -.093 & .014 & .884 & .009 & 0.766 & \\
\hline A6. Sensibilidad a los demás & .046 & .016 & .029 & .908 & .041 & 0.766 & \\
\hline Dominio de Escrupulosidad & & & & & & 0.855 & $11.68 \%$ \\
\hline ES1. Competencia & -.007 & .080 & .001 & -.001 & .806 & 0.568 & \\
\hline ES2. Orden & .069 & .002 & -.086 & .031 & .688 & 0.537 & \\
\hline ES3. Sentido del deber & -.041 & .075 & .075 & .003 & .735 & 0.590 & \\
\hline ES4. Necesidad de logro & -.041 & -.009 & -.031 & .030 & .774 & 0.497 & \\
\hline ES5. Autodisciplina & .036 & .037 & -.036 & -.087 & .781 & 0.501 & \\
\hline ES6. Deliberación & .041 & -.025 & .060 & .106 & .779 & 0.563 & \\
\hline Modelo completo & & & & & & 0.807 & $68.83 \%$ \\
\hline
\end{tabular}

En este análisis, las 30 facetas presentaron cargas factoriales superiores a 0.4 en los dominios de neuroticismo $(N)$, extraversión (EX), apertura a la experiencia $(A E)$, amabilidad $(A)$ y escrupulosidad $(E S)$. A raíz de estos hallazgos, fue posible determinar que la estructura del cuestionario NEO-PI-R utilizada en este estudio reproduce fielmente la estructura del cuestionario en su versión en inglés. La consistencia interna de las escalas fue evaluada por medio del coeficiente alfa de Cronbach. De acuerdo a la literatura, valores superiores a 0.7 se consideran suficientes para garantizar la fiabilidad de la escala. Los coeficientes alfa para las escalas de facetas individuales fueron inferiores a los de los dominios y, en algunos casos, menores a lo exigido en la literatura. No obstante, en la mayoría de los casos se demostró que eran valores aceptables para las escalas que tenían únicamente ocho ítems en comparación con los 48 ítems utilizados para cada uno de los cinco dominios. En el análisis de cada dominio, los resultados confirmaron la fiabilidad de la escala empleada.

\section{Liderazgo Transformacional}

Este apartado exhibe un análisis de estadística descriptiva y análisis factorial exploratorio del estilo de liderazgo transformacional. La Tabla 3 reporta los resultados derivados de los datos obtenidos del cuestionario 
MLQ. De acuerdo a Avolio y Bass (2004), el estilo de liderazgo transformacional está compuesto por cinco factores: (a) atributos de influencia idealizada, (b) comportamientos de influencia idealizada, (c) motivación inspiradora, (d) estimulación intelectual y (e) consideración individualizada. Las medias de cada factor se obtuvieron calculando el promedio de las puntuaciones de los ítems que la componen. Por otra parte, las medias del estilo de liderazgo transformacional se obtuvieron promediando las puntuaciones de cada factor.

Tabla 3: Estadística Descriptiva del Estilo de Liderazgo Transformacional

\begin{tabular}{|l|c|c|c|c|c|c|c|}
\hline Estilos de liderazgo & Media & D.E. & Mínimo & Máximo & Asimetría & Curtosis & $\begin{array}{c}\text { Coef. de } \\
\text { Variación }\end{array}$ \\
\hline Influencia idealizada (atributo) & 2.75 & 0.81 & 0.25 & 4.00 & -0.77 & 0.57 & 0.0022 \\
\hline Influencia idealizada (comportamiento) & 3.08 & 0.69 & 1.00 & 4.00 & -0.36 & -0.71 & 0.0019 \\
\hline Motivación inspiradora & 3.34 & 0.60 & 1.25 & 4.00 & -0.77 & -0.10 & 0.0016 \\
\hline Estimulación intelectual & 3.08 & 0.65 & 0.75 & 4.00 & -0.44 & -0.22 & 0.0018 \\
\hline Consideración individual & 3.01 & 0.71 & 0.75 & 4.00 & -0.24 & -0.63 & 0.0019 \\
\hline L. Transformacional & 3.05 & 0.55 & 0.90 & 4.00 & -0.55 & 0.26 & 0.0015 \\
\hline
\end{tabular}

Posteriormente se realizó un análisis factorial para establecer si los cinco factores podían reducirse al estilo de liderazgo transformacional. Para ello, la medida de adecuación muestral de Kaiser-Meyer-Olkin (KMO) de 0.761 y la prueba de esfericidad de Bartlett con un estadístico $x^{2}$ de $1239.59(p<0.01)$ fueron calculadas, denotando que se cumplen las condiciones para el uso del análisis factorial exploratorio (AFE). De acuerdo al método de análisis de componentes principales, los cinco factores explicaron el $63.34 \%$ de la varianza total. Se destaca además que los cinco factores presentaron cargas factoriales superiores a 0.4 (ver Tabla 4). Por último, la consistencia interna de las escalas fue evaluada por medio del coeficiente alfa de Cronbach. El coeficiente alfa para el puntaje de liderazgo transformacional fue de 0.924 cuando se evaluaron los 20 ítems de los cinco factores que componen el estilo de liderazgo. Los resultados confirmaron la fiabilidad de la escala empleada.

Tabla 4: Cargas Factoriales del Estilo de Liderazgo Transformacional

\begin{tabular}{|l|c|c|c|}
\hline \multicolumn{1}{|c|}{ Factor } & Factor 1 & $\alpha$ de Cronbach & Varianza explicada \\
\hline Liderazgo transformacional & & 0.924 & $63.34 \%$ \\
\hline Influencia idealizada (atributo) & .849 & 0.786 & \\
\hline Influencia idealizada (comportamiento) & .641 & 0.846 & \\
\hline Motivación inspiradora & .828 & 0.833 & \\
\hline Estimulación intelectual & .699 & 0.828 & \\
\hline Consideración individual & .927 & 0.841 & \\
\hline
\end{tabular}

\section{Relación entre factores}

El análisis de correlaciones fue realizado para determinar la fuerza de relación entre los cinco rasgos de personalidad y el estilo de liderazgo transformacional, por medio del coeficiente de correlación de Pearson. Cuando este coeficiente es más cercano a 1 en valor absoluto, existe mayor fuerza de relación. Por otro lado, coeficientes cercanos a cero en valor absoluto denotan una pobre relación lineal entre variables (Hair et al., 1999; Lind et al., 2012).

Para efectos de este análisis se utilizó como insumo los puntajes de los factores rotados con la técnica de rotación varimax y el promedio del estilo de liderazgo transformacional. La Tabla 5 reporta los resultados del análisis de correlaciones y los coeficientes significativos a un nivel de confianza de $99 \%$ han sido marcados con dos asteriscos $(p<0.01)$. La asociación positiva más fuerte del estilo de liderazgo transformacional se encontró con respecto a la extraversión y seguida de la escrupulosidad. Se observó, además, una asociación positiva relativamente débil con respecto a la apertura a la experiencia y una asociación negativa relativamente débil con respecto al neuroticismo. La amabilidad no presentó relación significativa en el análisis. Los resultados sugieren la posibilidad de usar un puntaje único para el liderazgo transformacional, encontrando relaciones significativas. 
Tabla 5: Correlaciones entre el Liderazgo Transformacional y los Dominios de Personalidad

\begin{tabular}{|l|c|c|c|c|c|}
\hline & Neuroticismo & Extraversión & $\begin{array}{c}\text { Apertura a la } \\
\text { experiencia }\end{array}$ & Amabilidad & Escrupulosidad \\
\hline L. Transformacional & $-.199^{* *}$ & $.289^{* *}$ & $.141^{* *}$ & -0.061 & $.273^{* *}$ \\
\hline
\end{tabular}

La Tabla 6 exhibe el modelo de regresión multivariado donde las variables exógenas son los cinco dominios de la personalidad más la experiencia laboral y la variable endógena es el estilo de liderazgo transformacional. Los valores de dominios de personalidad corresponden a las cargas factoriales de la rotación varimax para reducir problemas de multicolinealidad. Los coeficientes fueron marcados con tres, dos y un asterisco que representan significancia estadística a un nivel $p<0.01, p<0.05$ y $p<0.10$, respectivamente.

Tabla 6: Coeficientes de Regresión del Estilo de Liderazgo Transformacional

\begin{tabular}{|c|c|c|c|c|c|c|}
\hline \multirow[t]{2}{*}{ Modelo } & \multicolumn{2}{|c|}{$\begin{array}{l}\text { Coeficientes no } \\
\text { estandarizados }\end{array}$} & \multirow{2}{*}{$\begin{array}{c}\begin{array}{c}\text { Coeficientes } \\
\text { estandarizados }\end{array} \\
\beta \\
\end{array}$} & \multirow[t]{2}{*}{$t$} & \multirow[t]{2}{*}{ Sig. } & \multirow[t]{2}{*}{ FIV } \\
\hline & $\beta$ & E.E. & & & & \\
\hline (Constante) & 2.941 & .054 & - & 54.014 & $<0.001$ & - \\
\hline Amabilidad & -.037 & .025 & -.068 & -1.467 & .143 & 1.005 \\
\hline Apertura a la experiencia & .076 & .025 & $.138^{\star \star \star}$ & 2.995 & .003 & 1.001 \\
\hline Extraversión & .147 & .026 & $.269^{\star \star \star}$ & 5.734 & $<0.001$ & 1.034 \\
\hline Neuroticismo & -.110 & .025 & $-.200^{\star * *}$ & -4.342 & $<0.001$ & 1.000 \\
\hline Escrupulosidad & .130 & .027 & $.238^{\star * \star}$ & 4.909 & $<0.001$ & 1.106 \\
\hline Experiencia & .013 & .006 & $.113^{\star *}$ & 2.293 & .022 & 1.145 \\
\hline $\mathrm{R}^{2}$ ajustado & \multicolumn{2}{|c|}{0.481} & Prueba F & $18.143^{* * *}$ & Durbin-Watson & 1.990 \\
\hline
\end{tabular}

Con un nivel de significancia de $1 \%$, el coeficiente estandarizado con el efecto más importante fue el de la extraversión (0.269), seguido de la escrupulosidad (0.238) y el efecto negativo del neuroticismo $(-0.200)$. La apertura a la experiencia también mostró un efecto positivo en el estilo de liderazgo transformacional (0.138). La variable experiencia laboral también presentó un efecto significativo al 95\% de nivel de confianza. El único efecto no significativo fue del dominio de la amabilidad. El valor $\mathrm{p}$ para determinar la significancia de las pruebas $\mathrm{F}$ del modelo fue menor que 0.01 , asegurando que los coeficientes son distintos de cero. Por otro lado, el valor del coeficiente de determinación ajustado $\mathrm{R}^{2}$ reveló que el modelo explica aproximadamente el $48.1 \%$ de la variación del estilo de liderazgo transformacional. Los valores de factor de inflación de la varianza (FIV) y Durbin-Watson se obtuvieron para determinar problemas de multicolinealidad y autocorrelación de los residuos. La literatura sugiere que valores FIV mayores que 10 denotan problemas de multicolinealidad y que valores aproximados a 2 en el estadístico Durbin-Watson disminuyen la probabilidad de padecer de problemas de autocorrelación (Gujarati y Porter, 2010). Con base en estas aseveraciones, los resultados obtenidos no presentaron problemas de multicolinealidad y autocorrelación de los residuos.

\section{Comprobación de Hipótesis y Hallazgos}

En el presente estudio se planteó una hipótesis general que establece una relación funcional entre los rasgos de la personalidad y los comportamientos de liderazgo transformacional. La tabla 7 resume los resultados que permiten evaluar las sub-hipótesis que se derivan de la hipótesis general. Con respecto a las primeras seis hipótesis que tratan de la relación bivariada entre cada dominio de la personalidad con el estilo de liderazgo transformacional, los resultados evidencian una relación negativa y significativa entre el neuroticismo y el estilo de liderazgo transformacional y una relación positiva significativa del liderazgo transformacional con respecto a la extroversión, apertura a la experiencia, escrupulosidad y la experiencia laboral. Los resultados además determinaron que el dominio de amabilidad no presentó un efecto estadísticamente significativo en el estilo de liderazgo transformacional. La sub-hipótesis 7 fue parcialmente aceptada, dado que sólo los dominios de la personalidad del neuroticismo, la extraversión, la apertura a la experiencia y la escrupulosidad, además de la experiencia laboral fueron las variables independientes con efectos significativos en el estilo de liderazgo transformacional. La amabilidad no fue significativa. 
Tabla 7: Comprobación de Hipótesis de Estudio

\begin{tabular}{|c|c|c|c|c|}
\hline \multicolumn{2}{|r|}{ Hipótesis } & B estimado & $p$ & Observación \\
\hline $\mathrm{H}_{1}$ & \multicolumn{4}{|c|}{$\begin{array}{l}\text { Existe una relación funcional entre los rasgos de personalidad como un constructo o conjunto de } \\
\text { rasgos y los comportamientos de liderazgo transformacional y transaccional }\end{array}$} \\
\hline Neuroticismo & $\begin{array}{l}\mathrm{H}_{1.1} \text { : Existe una relación negativa entre el } \\
\text { neuroticismo y el liderazgo transformacional }\end{array}$ & -0.200 & $\star \star \star *$ & Aceptada \\
\hline Extraversión & $\begin{array}{l}\mathrm{H}_{1.2} \text { : Existe una relación positiva entre la } \\
\text { extraversión y el liderazgo transformacional }\end{array}$ & 0.269 & $* * *$ & Aceptada \\
\hline $\begin{array}{l}\text { Apertura a la } \\
\text { experiencia }\end{array}$ & $\begin{array}{l}\mathrm{H}_{1.3} \text { : Existe una relación positiva entre la } \\
\text { apertura a la experiencia y el liderazgo } \\
\text { transformacional }\end{array}$ & 0.138 & $* * *$ & Aceptada \\
\hline Amabilidad & $\begin{array}{l}\mathrm{H}_{1.4} \text { : Existe una relación positiva entre la } \\
\text { amabilidad y el liderazgo transformacional }\end{array}$ & -0.068 & - & Rechazada \\
\hline Escrupulosidad & $\begin{array}{l}\mathrm{H}_{1.5}: \text { Existe una relación positiva entre la } \\
\text { escrupulosidad y el liderazgo transformacional }\end{array}$ & 0.238 & $* \star *$ & Aceptada \\
\hline $\begin{array}{l}\text { Experiencia } \\
\text { laboral }\end{array}$ & $\begin{array}{l}\mathrm{H}_{1.6} \text { : Existe una relación positiva entre la } \\
\text { experiencia laboral y el liderazgo } \\
\text { transformacional }\end{array}$ & 0.113 & ** & Aceptada \\
\hline \multirow{6}{*}{$\begin{array}{l}\text { Dominios de } \\
\text { Personalidad }\end{array}$} & \multirow{6}{*}{$\begin{array}{l}\mathrm{H}_{1.7} \text { : Los cinco dominios de la personalidad, es } \\
\text { decir, neuroticismo, extraversión, apertura a la } \\
\text { experiencia, amabilidad y escrupulosidad, y } \\
\text { experiencia de trabajo ejercen una influencia } \\
\text { combinada sobre el estilo de liderazgo } \\
\text { transformacional }\end{array}$} & $\mathrm{N}=-.200$ & $\star \star *$ & \multirow{6}{*}{$\begin{array}{l}\text { Parcialmente } \\
\text { aceptada }\end{array}$} \\
\hline & & $E X=.269$ & $\star * \star$ & \\
\hline & & $\mathrm{AE}=.138$ & 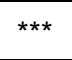 & \\
\hline & & $A=-.068$ & - & \\
\hline & & $E S=.238$ & $\star \star \star *$ & \\
\hline & & Experiencia $=.113$ & $* *$ & \\
\hline
\end{tabular}

Los resultados destacan que la extraversión y la escrupulosidad son los rasgos de la personalidad más consistentes del modelo. Esto demuestra que las personas sociables y dominantes, es decir, con un alto nivel de extraversión, poseen más probabilidades de afirmarse en situaciones grupales. El mismo escenario ocurre en la escrupulosidad, donde las personas conscientes pueden emerger rápidamente como líderes. Dichos hallazgos están ampliamente corroborados en la literatura (Bono y Judge, 2004; Do y Minbashian, 2014; Judge y Bono, 2000; Zareen et al., 2015; Zopiatis y Constanti, 2012). La apertura a la experiencia también reportó un coeficiente significativo y positivo, evidenciando que dicho rasgo representa una característica de líder transformacional. De hecho, la apertura a la experiencia describe una tendencia a ser creativo, imaginativo y curioso, permitiendo de esta forma que los gerentes muestren interés en descubrir nuevas oportunidades gerenciales y condiciones visionarias para el futuro de sus organizaciones (Bono y Judge, 2004).

Por otro lado, la amabilidad no presentó un coeficiente significativo para el contexto ecuatoriano, comprobando así los hallazgos de Judge et al. (2002), quienes identificaron a la amabilidad como el rasgo menos relevante del modelo de los Cinco Grandes, aseverando que los individuos agradables tienden a ser pasivos y obedientes y, por tanto, son menos propensos a emerger como líderes. El rasgo de neuroticismo mostró una relación moderadamente negativa con el estilo de liderazgo transformacional. Este hallazgo concuerda con estudios previos que sugieren que un líder requiere ser emocionalmente estable y un bajo nivel de neuroticismo es un importante predictor del potencial de liderazgo. Además, es poco probable que personas neuróticas demuestren rasgos de motivación, inspiración y estimulación intelectual (Bono y Judge, 2004; Costa y McCrae, 1992; McCrae y Costa, 1987). Se destacan varias implicancias derivadas de los hallazgos del estudio. A partir de los resultados, las empresas pueden establecer procesos de selección más eficientes al tomar en cuenta aspectos como la personalidad para puestos gerenciales o de mandos medios y altos. A mediano plazo, los resultados pueden aportar en la producción de condiciones óptimas a nivel individual que promuevan las probabilidades de desarrollo de las características deseadas de liderazgo, de manera que la selección de personal para cargos administrativos en el contexto ecuatoriano sea óptima.

\section{CONCLUSIONES}

A raíz de los resultados, se plantean las siguientes conclusiones: 1 . El presente estudio contribuye con nuevos conocimientos del liderazgo transformacional debido a que presenta nuevas evidencias del efecto de los cinco principales dominios de la personalidad en este estilo de liderazgo; 2. Dominios como la extraversión y la 
escrupulosidad demostraron ser los más importantes en la proyección del estilo de liderazgo, pues las personas con altos niveles de dichos rasgos son más propensas a presentar comportamientos de líderes transformacionales; 3 . Los resultados señalan también que la apertura a la experiencia tiene una relación positiva y significativa estadísticamente con el estilo de liderazgo transformacional; 4. El dominio de amabilidad no presentó un efecto estadísticamente significativo en los estilos de liderazgo transformacional, consistente con otros hallazgos en la literatura; 5 . Los años de experiencia laboral influyen positivamente en la presencia de comportamientos de liderazgo transformacional.

\section{REFERENCIAS}

Araneda, C., N. Neumann, L. Pedraja y E. Rodríguez, Análisis Exploratorio de las Percepciones sobre los Estilos de Liderazgo de los Directivos Universitarios en el Norte de Chile, Formación Universitaria, 9(6), 139-152 (2016)

Arora, R. y S. Rangnekar, The interactive effects of conscientiousness and agreeableness on career commitment, Journal of Employment Counseling, 53(1), 14-29 (2016)

Avolio, B.J. y B. M. Bass, Multifactor Leadership Questionnaire: Manual and sampler test, $3^{\text {rd }}$ Ed., Redwood City, CA: Mind Garden (2004)

Ballester-Miquel, J.C., P. Pérez-Ruiz, J. Hernández-Gadea y D. Palacios-Marqués, Implementation of the Balanced Scorecard in the Hotel Sector through Transformational Leadership and Empowerment, Multidisciplinary Journal for Education, Social and Technological Sciences, 4(1), 1-15 (2017)

Bass, B.M., The Bass handbook of leadership: Theory, research and managerial applications, $4^{\mathrm{a}}$ Ed., New York, Free Press (2008)

Bass, B. M. y B. J. Avolio, Improving organizational effectiveness through transformational leadership, Thousand Oaks, CA, Sage (1994)

Boe, O. y T. Holth, The relationship between developmental leadership, the results of leadership and personality factors, Procedia Economics and Finance, 26, 849-858 (2015)

Bono, J.E. y T.A. Judge, Personality and transformational and transactional leadership: a meta-analysis, Journal of Applied Psychology, 89(5), 901-910 (2004)

Bono, J., A. Hooper y D. Yoon, Impact of rater personality on transformational and transactional leadership ratings, The Leadership Quarterly, 23(1), 132-145 (2012)

Burns, J.M., Leadership, New York, Harper y Row (1978)

Costa, P.T. y R.R. McCrae, Four ways five factors are basic, Personality and individual differences, 13(6), $653-665$ (1992)

Crossan, M., D. Mazutis, G. Seijts, y J. Gandz, Developing Leadership Character in Business Programs, Academy of Management Learning \& Education, 12(2), 285-305 (2012)

D'Alessio, F., The influence of Personality Domains and Working Experience in Peruvian Manager's Leadership Styles: An initial study, Journal of CENTRUM Cathedra, 13-33 (2008)

Do, M. y A. Minbashian, A meta-analytic examination of the effects of the agentic and affiliative aspects of extraversion on leadership outcomes, The Leadership Quarterly, 25(5), 1040-1053 (2014)

Furnham, A. y J. Crump, Personality and Management Level: Traits That Differentiate Leadership Levels, Psychology, 6 , 549-559 (2015)

Goldberg, L.R., An alternative "description of personality": The Big-Five factor structure, Journal of Personality and Social Psychology, 59, 1216-1229 (1990)

Gujarati, D., y D. Porter, Econometría, 5a Ed., México D.F., McGraw-Hill (2010)

Hair, J., R. Anderson, R. Tatham y W. Black, Análisis Multivariante, Madrid, Prentice Hall (1999)

Hezlett, S. A., Enhancing Experience-Driven Leadership Development, Advances in Developing Human Resources, 18(3), 369-389 (2016)

Hofstede, G., Culture's consequences - Comparing values, behaviors, institutions, and organizations across nations, $2^{\text {nd }}$ Ed., Newbury Park, Sage (2001)

Hofstede, G., 'What did GLOBE really measure? Researchers' minds versus Respondents' minds', Journal of International Business Studies, 37(6), 882-96 (2006)

Johnson, S. K. y R. L. Dipboye, Effects of charismatic content and delivery on follower task performance: The moderating role of task charisma conduciveness, Group \& Organization Management, 33(1), 77-106 (2008)

Judge, T.A. y J.E. Bono, Five-Factor Model of Personality and Transformational Leadership, Journal of Applied Psychology, 85, 751-765 (2000)

Judge, T.A., J.E. Bono, R. Ilies y M. W. Gerhardt, Personality and leadership: a qualitative and quantitative review, Journal of applied psychology, 87(4), 765 (2002) 
Judge, T.A., A. Erez, J.E. Bono y C. J. Thoresen, Are measures of self-esteem, neuroticism, locus of control, and generalized self-efficacy indicators of a common core construct? Journal of Personality and Social Psychology, 83(3), 693710 (2002)

Kaufman, S.B., L.C. Quilty y otros cinco autores, Openness to experience and intellect differentially predict creative achievement in the arts and sciences, Journal of Personality, 84(2), 248 (2016)

Lim, B.C. y R. E. Ployhart, Transformational leadership: relations to the five-factor model and team performance in typical and maximum contexts, Journal of Applied Psychology, 89(4), 610 (2004)

Lind, D., W. Marchal y S. Wathen, Estadística aplicada a los negocios y la economía, 15ª Ed., México, McGraw-Hill (2012) McAdams, K.K. y M.B. Donnellan, Facets of personality and drinking in first-year college students. Personality and Individual Differences, 46(2), 207-212 (2009)

McCall Jr, M. W. y G. P. Hollenbeck, Developing global executives: The lessons of international experience. Boston, MA: Harvard Business School Press (2002)

McCall Jr, M.W. y C.D. McCauley, Experience-Driven Leadership Development. En Using Experience to Develop Leadership Talent, 1-15, (2014)

McCrae, R.R. y P. T. Costa, Validation of the five-factor model of personality across instruments and observers, Journal of personality and social psychology, 52(1), 81 (1987)

Sistema Nacional de Información de Educación Superior del Ecuador. Geoportal SNIESE. Obtenido de Oferta académica vigente en el Ecuador (2017)

Soto, C.J., Is happiness good for your personality? Concurrent and prospective relations of the big five with subjective well-being, Journal of Personality, 83(1), 45-55 (2015)

Spinelli, R.J., The applicability of Bass's model of transformational, transactional, and laissez-faire leadership in the hospital administrative environment, Hospital Topics, 84(2), 11-19 (2006)

Tanasescu, V., C. B. Jones y otros cuatro autores, The Personality of Venues: Places and the Five-Factors ('Big Five') Model of Personality, 2013 Fourth International Conference on IEEE., 76-81, Computing for Geospatial Research and Application (2013)

Thompson, B., Exploratory and confirmatory factor analysis: Understanding concepts and applications. Washington, DC, American Psychological Association (2004)

Torres, D., y S. Jones, A changing scene: Comparing business cultures in Peru and the Netherlands. Wiley Periodicals Inc., 53-66 (2010)

Zareen, M., K. Razzaq y B. G. Mujtaba, Impact of transactional, transformational and laissez-faire leadership styles on motivation: A quantitative study of banking employees in Pakistan, Public Organization Review, 15(4), $531-549$ (2015)

Zopiatis, A. y P. Constanti, Extraversion, openness and conscientiousness: The route to transformational leadership in the hotel industry, Leadership \& Organization Development Journal, 33(1), 86-104 (2012) 